\title{
Hemodynamic Impairment Measured by Positron-Emission Tomography Is Regionally Associated with Decreased Cortical Thickness in Moyamoya Phenomenon
}

\author{
(D).J. Lee, (D).S. Shimony, (DH. Jafri, (D)A.R. Zazulia, (D).G. Dacey, Jr., (D) G.R. Zipfel, and (DC.P. Derdeyn
}

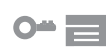

\begin{abstract}
BACKGROUND AND PURPOSE: Impaired cerebrovascular reactivity has been associated with decreased cortical thickness in patients with arterial occlusive diseases. This study tests the hypothesis that severe hemodynamic impairment, indicated by increased oxygen extraction fraction ratios on positron-emission tomography with ${ }^{15} \mathrm{O}$ tracers, is associated with decreased cortical thickness in patients with Moyamoya phenomenon.
\end{abstract}

MATERIALS AND METHODS: Patients with unilateral or bilateral idiopathic Moyamoya phenomenon were recruited. Oxygen extraction fraction ratio maps were generated from cerebral images of $\mathrm{O}\left[{ }^{15} \mathrm{O}\right]$ counts divided by $\mathrm{H}_{2}\left[{ }^{[5} \mathrm{O}\right]$ counts with normalization by corresponding cerebellar counts. The normal range of the oxygen extraction fraction ratio was estimated from historically available healthy control subjects. Cortical thickness was estimated from T1-weighted MR imaging and FreeSurfer. Regional samples of oxygen extraction fraction ratios and cortical thicknesses were drawn using FreeSurfer parcellations, retaining only parcellations from the vascular territory of the middle cerebral artery.

RESULTS: Complete MR imaging and PET datasets were available in 35 subjects, including 23 women; the mean age at scanning was 44 years. Patients with Moyamoya phenomenon had a significantly increased regional oxygen extraction fraction ratio compared with 15 healthy control subjects $(P<.001)$. Regional oxygen extraction fraction ratio and age were significant predictors of cortical thickness $(P<$ .001 for each) in a generalized linear mixed-effects model. Using hemisphere averages and patient averages, we found that only age was a significant predictor of cortical thickness $(P<.001)$.

CONCLUSIONS: Chronic hemodynamic impairment, as indicated by a higher regional oxygen extraction fraction ratio, was significantly predictive of reduced cortical thickness in mixed-effects analysis of FreeSurfer regions. This phenomenon may be related to reversible metabolic down-regulation.

ABBREVIATIONS: OEF = oxygen extraction fraction; OEFR = oxygen extraction fraction ratio; SEM = standard error of measurement

hronic hemodynamic impairment from basal artery occlusive disease may lead to regional reductions in cortical thickness. Fierstra et $\mathrm{al}^{1}$ examined patients with unilateral occlusive

Received February 12, 2018; accepted after revision August 6.

From the Mallinckrodt Institute of Radiology (J.J.L., J.S.S., H.J., A.R.Z.) and Departments of Neurology (A.R.Z., G.R.Z.) and Neurosurgery (R.G.D., G.R.Z.), Washington University, St Louis, Missouri; and Department of Radiology (C.P.D.), University of lowa, lowa City, lowa.

This work was supported by the National Institute of Neurological Disorders and Stroke RO1 NS051631 (C.P.D.). Support for the research imaging center where the MR studies were performed was provided by UL1 RR024992-01, TL1 RR024995-01, and KL2 RR 024994-01 from the National Center for Research Resources, a component of the National Institutes of Health, and the National Center for Research Resources Roadmap for Medical Research, P30NS048056. J.S.S. was also supported by the Eunice Kennedy Shriver National Institute of Child Health \& Human Development of the National Institutes of Health under Award Number U54 HD087011 to the Intellectual and Developmental Disabilities Research Center at Washington University. cerebrovascular disease using $\mathrm{CO}_{2}$ reactivity and diminished blood oxygen level-dependent responses as a marker for regional hemodynamic impairment. They demonstrated that regions with steal physiology and a reduced blood oxygen level-dependent response to $\mathrm{CO}_{2}$ had cortical thickness $(2.40 \pm 0.03 \mathrm{~mm}$ standard error of measurement [SEM]) that increased $5.1 \%$ after successful surgical revascularization $(2.53 \pm 0.03 \mathrm{~mm} \mathrm{SEM})$.

The content of this work is solely the responsibility of the authors and does not necessarily represent the official view of the National Center for Research Resources or the National Institutes of Health.

Clinical trial registration information: http://www.clinicaltrials.gov. Unique identifier: NCT00629915.

Please address correspondence to John J. Lee, MD, PhD, Mallinckrodt Institute of Radiology, Washington University School of Medicine, 4525 Scott Ave, Campus Box 8225, St Louis, MO 63110; e-mail: jjlee@wustl.edu; @jjleeWustlEdu

- Indicates open access to non-subscribers at www.ajnr.org

三 Indicates article with supplemental on-line tables.

http://dx.doi.org/10.3174/ajnr.A5812 
Methods to categorize hemodynamic impairment are based on the response of the cerebrovasculature to vasodilatory challenges, measurements of increased blood volume, mean transit time, or measurements of an increased oxygen extraction fraction (OEF). ${ }^{2}$ The diminished blood oxygen level-dependent response to $\mathrm{CO}_{2}$ is likely a response of pre-existing autoregulatory vasodilation in response to reduced perfusion pressure. ${ }^{2}$ OEF measurement uses ${ }^{15} \mathrm{O}$ radiotracers to quantify oxygen extracted from blood by the brain. When cerebral blood flow falls, the OEF can increase from a normal baseline of $40 \%$ up to $80 \%$ to maintain normal oxygen metabolism and brain function. ${ }^{3,4}$ Increased OEF has been shown to be a powerful predictor of stroke in patients with atherosclerotic carotid artery occlusion. ${ }^{2,5-7}$

Quantitative OEF requires radial artery cannulation, multiple levels of calibrations, and the assumption that first-passage of the bolus of radiolabels along the radial artery is an accurate proxy for first-passage of the bolus through the cerebral circulation. For chronic carotid occlusive disease, methods of OEF estimation using emission counts and normalization against reference values have had comparable predictive values for clinical outcomes and improved receiver operating characteristics. ${ }^{89}$ In this work, we used the ratio of count-based OEF (OEFR) in the cerebral regions to count-based OEF in the cerebellum for all analyses. ${ }^{10}$

This study sought to test the hypothesis that chronic hemodynamic impairment, as identified by an OEFR, is associated with decreased cortical thickness in patients with Moyamoya phenomenon. ${ }^{1}$

\section{MATERIALS AND METHODS \\ Patients}

Technically sufficient MR imaging, PET imaging, and clinical data were obtained from 35 patients enrolled in a prospective, blindly adjudicated study of hemodynamic factors and stroke risk. ${ }^{11}$ Inclusion criteria were unilateral or bilateral Moyamoya phenomenon by angiography. Patients with prior surgical revascularization were included if the contralateral hemisphere was affected and untreated or if there were recurrent symptoms in the treated hemisphere. In analyses, data were excluded if the encompassing hemisphere demonstrated cortical infarction or hemorrhage. These inclusion and exclusion criteria and their applicability to cerebral hemispheres as well as individual patients were based on criteria used in previous cortical thickness studies of Moyamoya phenomenon by Fierstra et al. ${ }^{1}$ On enrollment, patients traveled to Washington University Medical Center for a detailed neurologic history, physical examination, interviews for baseline demographics and stroke risk factors, MR imaging, and PET imaging.

The present data are a subset of data from a prospective natural history study by Derdeyn et al. ${ }^{11}$ Of the 50 subjects in the previous study, 15 were excluded from this study because of the following: technically incomplete imaging necessary for this study, bilateral infarctions, bilateral hemorrhages, or surgical treatment with improvement. Methodologic differences between the prior study and this one included the following: use of FreeSurfer (http://surfer.nmr.mgh.harvard.edu) ${ }^{12-14}$ automated cortical parcellations, classification of regions most likely to be in the
MCA vascular territory, granular identification of regional peaks of OEFR, and use of generalized linear mixed models.

Solely for purposes of estimating the range of normal values for OEFR, technically sufficient, historically available PET data from Grubb et $\mathrm{al}^{6}$ were gathered from 15 subjects, including 8 women, who had previously been recruited for healthy control PET measurements; the mean age for this cohort was $48 \pm 18$ years. All control subjects had normal neurologic examination findings and normal brain MR imaging findings. Cortical thickness assessments were not technically feasible in the historical data. Written, informed consent was obtained for all patients and healthy subjects according to guidelines of the Washington University institutional review boards.

\section{PET Imaging}

All PET imaging was performed at a single ECAT EXACT HR+ scanner (Siemens, Erlangen, Germany). Scanning began with transmission acquisitions from $\left[{ }^{68} \mathrm{Ge}\right] /\left[{ }^{68} \mathrm{Ga}\right]$ sources for attenuation correction. Subjects inhaled 1 or 2 breaths from tubing connected to a shielded canister containing room air and $100 \mathrm{mCi}$ (nominal) of $\mathrm{O}\left[{ }^{15} \mathrm{O}\right]$. Emission scanning began simultaneously, lasting 120 seconds. After waiting at least 15 minutes, a bolus of 75 mCi (nominal) of $\mathrm{H}_{2}\left[{ }^{15} \mathrm{O}\right]$ was injected through an antecubital intravenous cannula, and emissions were scanned for $120 \mathrm{sec}-$ onds. Following filtered back-projection reconstruction with corrections for normalization, attenuation, and scatter, reconstructed images had $2.0 \times 2.0 \times 2.4 \mathrm{~mm}$ resolution in an FOV of $128 \times 128 \times 63$ voxels. Dynamic oxygen and water image frames were temporally summed over 40 seconds starting from the time of tracer arrival into the FOV; then they were Gaussian-filtered to the measured point-spread function of the scanner, $7.3 \times 7.3 \times$ $5.3 \mathrm{~mm}$ full width at half maximum. ${ }^{15,16}$

\section{MR Imaging}

All MR imaging studies used the same 3T system (Magnetom Tim Trio; Siemens). MR imaging was performed within 24 hours of PET scanning, in most cases within 1 hour after the conclusion of PET studies. All except 4 patients were scanned by MPRAGE series (TR, $2000 \mathrm{~ms}$; TE, $2.97 \mathrm{~ms}$ ) with a sagittal orientation, $1 \times 1 \times$ $1 \mathrm{~mm}$ resolution, and $208 \times 256 \times 144$ FOV. Some MPRAGE scans had $1.25 \times 1.25 \times 1.3 \mathrm{~mm}$ resolution. For detecting white matter lesions, FLAIR series (TR, $4000 \mathrm{~ms}$; TE, $294 \mathrm{~ms} ; 0.9 \times$ $0.9 \times 5.0 \mathrm{~mm}$ resolution; $256 \times 256 \times 26 \mathrm{FOV})$ and T2-weighted imaging (TR, $3200 \mathrm{~ms}$; TE, $319 \mathrm{~ms}$; turbo spin-echo; $0.45 \times$ $0.45 \times 5.0 \mathrm{~mm}$ resolution; $416 \times 512 \times 26 \mathrm{FOV}$ ) were also obtained.

\section{Analysis of OEFR and Cortical Thickness}

All ROIs made use of the Destrieux parcellations, aparc.a2009s + aseg, obtained from recon-all in FreeSurfer (Linux GNU stable, Version 5 20130513). ${ }^{12-14,17}$ Fully regional analyses used cortical regions numbered 11101-11175 and 12101-12175 and cerebellar regions 7,8 , 46, and 47 as discovered by recon-all and using additional selection criteria detailed below. The FMRIB Linear Image Registration Tool (FLIRT, Version 5; http://www.fmrib. ox.ac.uk/fsl/fslwiki/FLIRT) ${ }^{18}$ created coregistrations of all tracer studies from PET onto FreeSurfer-generated T1.mgz images us- 
ing mutual information cost functions. The mri_segstats application from FreeSurfer provided statistical results for all regions. The regions provided sampling of emission counts directly from $\mathrm{O}\left[{ }^{15} \mathrm{O}\right]$ and $\mathrm{H}_{2}\left[{ }^{15} \mathrm{O}\right]$, both cortical and cerebellar, and OEFRs were calculated from regional mean counts normalized by the corresponding measurements in the cerebellum. All cerebellar regions were bilaterally pooled according to patient identity to avoid possible artifacts from crossed cerebellar diaschisis.

To increase the specificity of analysis, we manually classified the Destrieux regions ${ }^{17}$ according to vascular territories previously reported by van der Zwan et al. ${ }^{19}$ van der Zwan et al had empirically determined estimates of the volumetric extent of the anterior, middle, and posterior cerebral arterial circulations by injecting colored Araldite F mixtures (Kirkside Products, Osborne Park, Western Australia) into arterial sources of unfixed human brains obtained at routine postmortem. The largest volumetric extent of the middle cerebral artery territory was identified by visual inspection with Destrieux parcellations on the Montreal Neurological Institute 305 atlas. By submitting a subset of Destrieux region numbers 11101-11175 and 12101-12175 to mri_segstats, we obtained estimates of OEFRs for 60 parcellations per hemisphere most likely to correspond to the MCA territories. All OEFR measurements were matched with cortical thickness measurements from identical, coregistered sampling volumes in the images; the correspondence was ensured throughout analyses of regions, hemispheres, and patients. The PET data from historical, healthy control subjects did not include high-resolution MPRAGE; consequently, the Montreal Neurological Institute 305 atlas was coregistered to the historical PET images, and mapped Destrieux regions were subsequently sampled to obtain OEFRs.

Analysis with granularity for hemispheres facilitates comparison of this work with the limited number of existing cortical thickness reports. Granularity for patient identity facilitates comparison with existing Moyamoya phenomenon case series. Thus, measurements made on Destrieux regions for MCA territories within a hemisphere were pooled by hemispheres and patient identities and analyzed separately.

All registration results and OEFR calculations were individually reviewed as maps coregistered to T1.mgz anatomy as well as surface inflation maps. Regions of cortical infarction noted in clinical histories and MR imaging were marked for exclusion from further analyses. Most artifacts identified in quality-control reviews were associated with infarcted areas previously identified by exclusion criteria. All processing pipelines and analyses were coded in Matlab (Mathworks, Natick, Massachusetts) for reproducibility.

\section{Statistical Analyses}

The historical healthy control data solely provided confidence intervals for estimating normal OEFR. $T$ tests for differences of regional OEFR between controls and patients with Moyamoya phenomenon were 2-tailed and heteroscedastic because these measurements were drawn from distinct studies, clinical categories, and subjects (ttest2 from Matlab R2017a). Generalized linear mixed-effects models used normal priors without link functions (fitglme from Matlab). Mixed-effect models appropriately describe the hierarchic covariance structures of measurements made by regions, hemispheres, and subjects and the repeated measurements necessarily made on hierarchic data. In this work, mixedeffects models tested the OEFR, age, and sex as predictors of cortical thickness. ${ }^{15,20}$ Intercepts, OEFR, age, and sex always had fixed effects. Intercepts and OEFR were explored using Akaike and Bayesian information criteria as random effects and grouped by patient identity to account for covariance structures between regions for a given patient. There were no other adjustments for multiple measurements. The significance level was always $95 \%$. Analyses computed joint covariances of fixed effects and covariance parameters and assessed the Hessian of the objective function at convergence. The maximum pseudolikelihood algorithm estimated model parameters with 10-100 iterations.

\section{RESULTS}

On-line Table 1 lists demographic, clinical, diagnostic, and treatment results both from Derdeyn et $\mathrm{al}^{11}$ and this study. The natural history study of 50 patients by Derdeyn et al used OEFRs in hemispheres as a biomarker. This study examined a subset of $35 \mathrm{pa}-$ tients with technically adequate imaging, after imposing additional inclusion and exclusion criteria described in the Materials and Methods, to enable FreeSurfer-based regional analyses. The elevated regional OEFR in this study is defined to exceed the upper 95\% confidence limit of regional OEFR measured in the control group of healthy subjects described in the Materials and Methods and quantified below. Characteristics listed in On-line Table 1 indicate the generalizability of these study results.

On-line Table 1 reports partitioning of patient census and measurements into unilateral and bilateral Moyamoya phenomenon in the category of vasculopathy on angiography. Of $10 \mathrm{pa}-$ tients with unilateral Moyamoya phenomenon, onlyl patient did not have an elevated regional OEFR; however, the hemisphere with Moyamoya phenomenon was excluded because of resolved symptoms after surgical revascularization. Of 25 patients with bilateral Moyamoya disease, 3 had an elevated regional OEFR.

Thirteen hemispheres in 13 patients were excluded from analyses in accordance with predetermined criteria, described in the Materials and Methods, applied to hemispheres. Of the remainder, 9 hemispheres in 9 patients were excluded for cortical infarction in the middle cerebral artery territory. Three hemispheres in 3 patients were excluded for basal ganglia hemorrhage. Three patients had prior surgical unilateral revascularization procedures. One of these revascularized hemispheres was excluded for basal ganglia hemorrhage; another hemisphere was excluded for absence of recurrent symptoms after surgical treatment. A total of 57 hemispheres in 35 patients were included in analyses.

\section{Imaging OEFR and Cortical Thickness}

Figure $1 A-D$ shows regional OEFRs fused with $\mathrm{T} 1$ anatomy and cortical thickness embedded in inflated surface maps for 4 patients randomly sampled from the patient cohort. Matlab function random-sampled patient identities following inclusion and exclusion selections. Higher regional OEFR was associated with a thinner cortex on visual inspection. However, venous sinuses and adjacent regions with partial volume averaging have artifactually elevated OEFRs arising from venous tracer activities because the AJNR Am J Neuroradiol 39:2037-44 Nov 2018 www.ajnr.org 


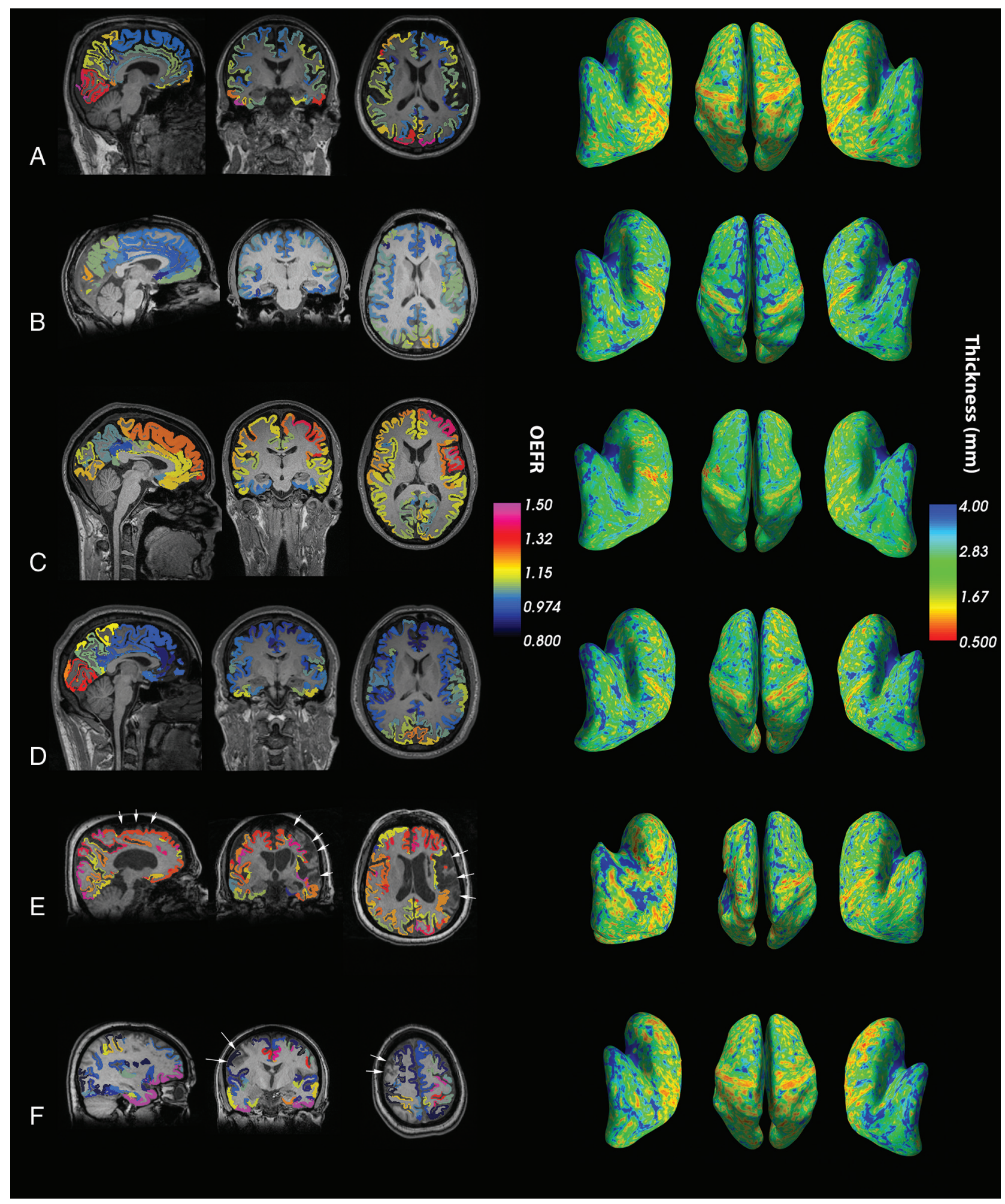

FIG 1. Representative views of OEFRs averaged over Destrieux parcellations, T7-anatomy, and cortical thickness on inflated surface maps. A-D, Four patients without infarction or hemorrhage were selected randomly from the patient cohort for visualization. E, This patient exemplifies the rationale for excluding infarcted hemispheres. Extensive encephalomalacia distorts cortical parcellations and confounds calculations on cortical and pial anatomy by FreeSurfer (arrows). F, This patient demonstrates paradoxically decreased OEFR around a region of infarction (arrows). The validity and significance of such decreases near infarcted areas are unknown. Consequently, all hemispheres ipsilateral to infarctions or hemorrhages were excluded. Modal color maps are identical for all subfigures and views.

OEFR method does not correct for cerebral blood volumes. Restricting analyses to MCA regions mitigates these artifacts. Figure $1 E,-F$ demonstrates confounding artifacts in hemispheres with infarction or hemorrhage. Anatomic pathology such as encephalomalacia presented challenges to the parcellation algorithms in FreeSurfer. Consequently, automated identifying of regions be- 

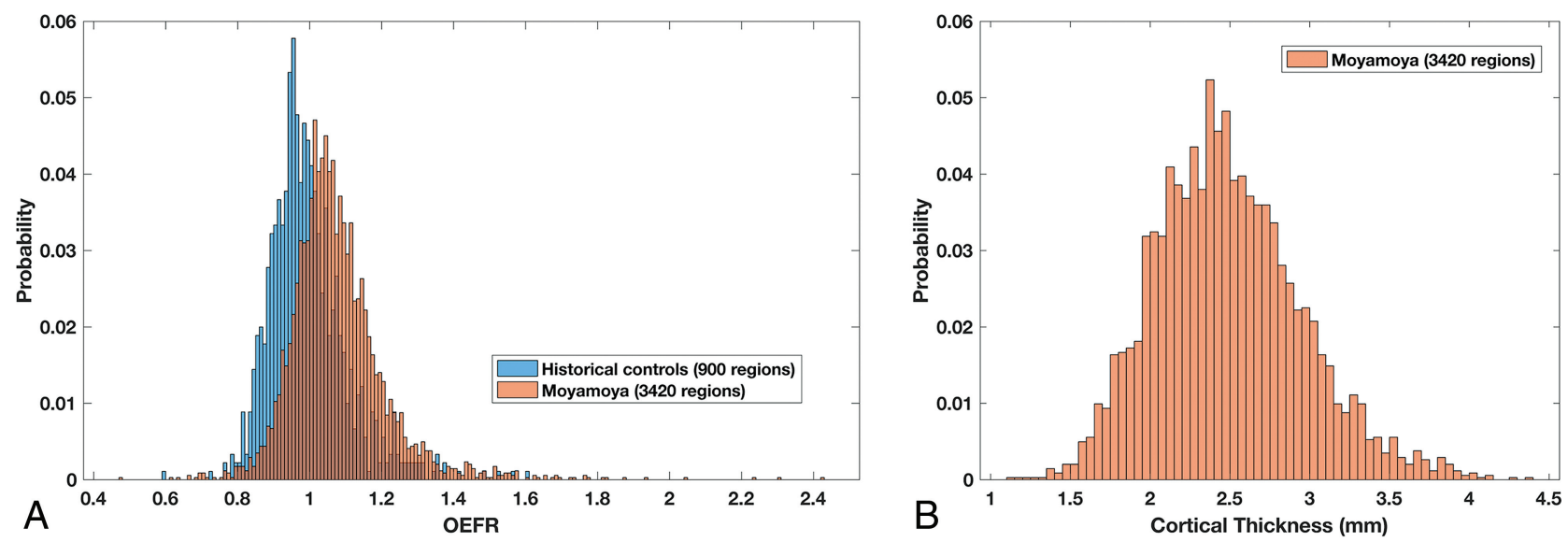

FIG 2. A, Histogram of OEFR from Destrieux FreeSurfer regions within MCA vascular territories from 15 historically obtained healthy control subjects. Superimposed is the histogram of OEFR from anatomically corresponding regions from 35 patients with Moyamoya phenomenon. Following applying exclusion criteria, there are 900 regions from controls and 3420 regions from patients. $B$, Histogram of cortical thickness from the same 3420 MCA regions in patients with Moyamoya phenomenon.

comes unreliable, and partial volume averaging with adjacent nonviable tissues made OEFR estimates uninterpretable.

\section{Distributions of OEFR and Cortical Thickness}

A historical dataset of 15 healthy subjects provided 900 Destrieux regions from MCA territories for use in estimating confidence intervals for the normal range of OEFRs. ${ }^{15}$ The distribution of OEFRs is shown in Fig $2 A$ in blue. The mean OEFR was 0.995 with 95\% CI, 0.776-1.213, and values above 1.213 were considered elevated. For patients with Moyamoya phenomenon, after inclusion and exclusion selections, 3420 Destrieux MCA regions had OEFRs distributed as shown in Fig $2 A$ in orange. The mean OEFR was 1.076 with $95 \%$ CI, $0.805-1.357$. The 2 -tailed $t$ test rejects the null hypothesis of equal means with unequal variances between healthy controls and patients with 95\% CI, 0.07-0.090. For all patients with Moyamoya phenomenon, the 3420 regions had cortical thicknesses distributed according to Fig $2 B$ with a mean of $2.49 \pm 0.01 \mathrm{~mm}$ SEM and 95\% CI, 1.57-3.41. There were 376 hemodynamically impaired Moyamoya regions with elevated OEFRs of $>1.213$; their cortical thicknesses had a mean of $2.34 \pm$ $0.02 \mathrm{~mm}$ SEM and 95\% CI, 1.47-3.21. On-line Table 1 enumerates how demographics, categoric clinical presentations, and angiographic confirmation of unilateral Moyamoya phenomena or bilateral Moyamoya disease varied with respect to OEFR and nominal elevations of OEFR.

\section{Mixed-Effects Models}

Figure 3 and On-line Table 2 detail results from generalized linear mixed-effects models. For measurements with granularity for Destrieux FreeSurfer regions within MCA territories, 3420 regions had OEFRs ranging from 0.479 to 2.43 ; age, 25-72 years; time from symptom onset to scanning from 0.1 to 87 months; and cortical thickness ranging from 1.10 to $4.38 \mathrm{~mm}$. The best model, reported in the Wilkinson Notation in On-line Table 2, had 5 fixed-effects coefficients and 70 random-effects coefficients for the intercept and OEFR for each patient grouping. The groupings described interhemispheric covariance patterns computed with 4 parameters in full Cholesky form. For the Destrieux FreeSurfer regions within MCA territories, OEFR and age were significant predictors of cortical thickness at the $5 \% \alpha$ level with $P<.001$. After we removed the OEFR and time from onset to scanning from the mixed model, only age was a significant predictor.

For measurements with granularity for hemispheres, $57 \mathrm{hemi-}$ spheres had OEFRs from 0.917 to 1.32 and cortical thickness from 1.94 to $2.81 \mathrm{~mm}$. The best model had 35 random-effects coefficients for the intercept for each patient grouping and an isotropic covariance pattern with 2 parameters. Only age was a significant predictor of cortical thickness, regardless of whether OEFR or time to scanning was included in the mixed model.

For measurements with granularity for patients ( 1 or 2 hemispheres), 35 patients had an OEFR from 0.932 to 1.32 and cortical thickness from 2.01 to $2.81 \mathrm{~mm}$. In numeric analyses, the data did not support any random effects. Only age was a significant predictor of cortical thickness regardless of inclusions of OEFR or time to scanning in the mixed model.

\section{DISCUSSION}

The present data support the observation that cortical thickness is reduced in chronic hemodynamic impairment. ${ }^{1,11,21}$ This study aimed to maintain continuity with the larger body of cortical thickness research, including the study of Moyamoya phenomenon by Fierstra et al. ${ }^{1,21}$ Patients with Moyamoya phenomenon from Fierstra et al, with steal phenomena before surgical revascularization, had a hemispheric mean thickness of $2.40 \pm 0.03 \mathrm{~mm}$ SEM. Patients with Moyamoya phenomenon in this study had FreeSurfer regions characterized by increased OEF, and their mean thickness was $2.34 \pm 0.02 \mathrm{~mm}$ SEM. However, this work differs from the prior studies of Fierstra et al, in 3 important ways. First, hemodynamic impairment in the previous studies was identified by regions that demonstrated paradoxically reduced blood oxygen level-dependent signal after $\mathrm{CO}_{2}$ challenge (steal phenomenon). ${ }^{1}$ Hemodynamic impairment in the present work was identified by increased OEFRs. These 2 physiologies are fundamentally different, though both likely reflect responses to reduced perfusion pressure or blood flow. ${ }^{2}$ Second, in the original 2010 study of Fierstra et al, all patients had unilateral occlusive disease, and the comparison was made between brain regions with steal 
FreeSurfer Regions
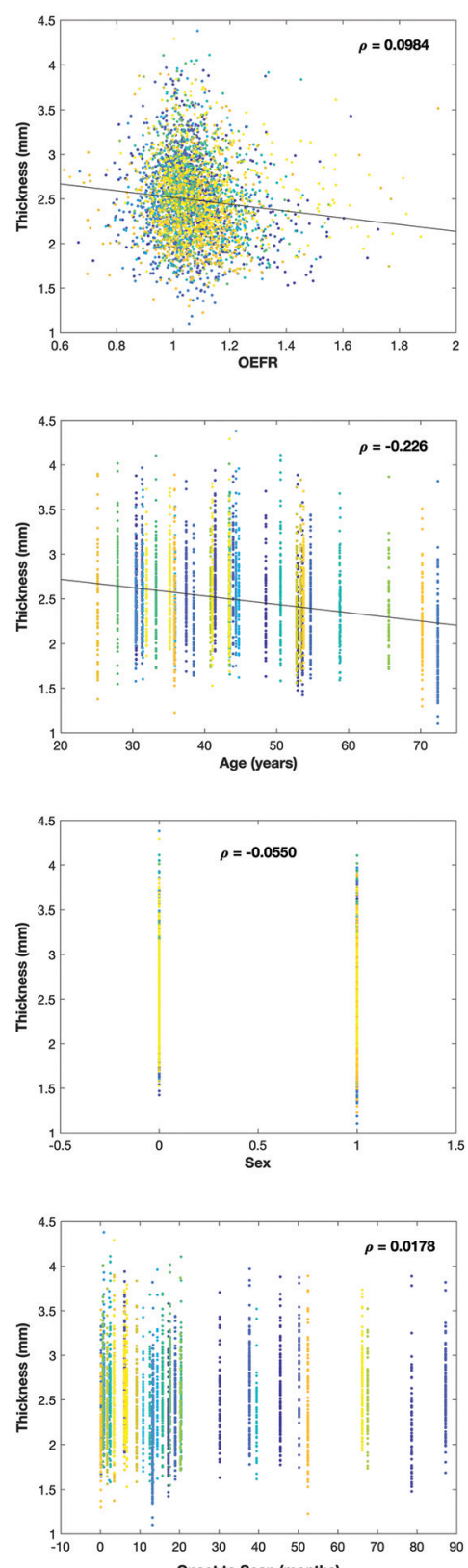

Hemispheres
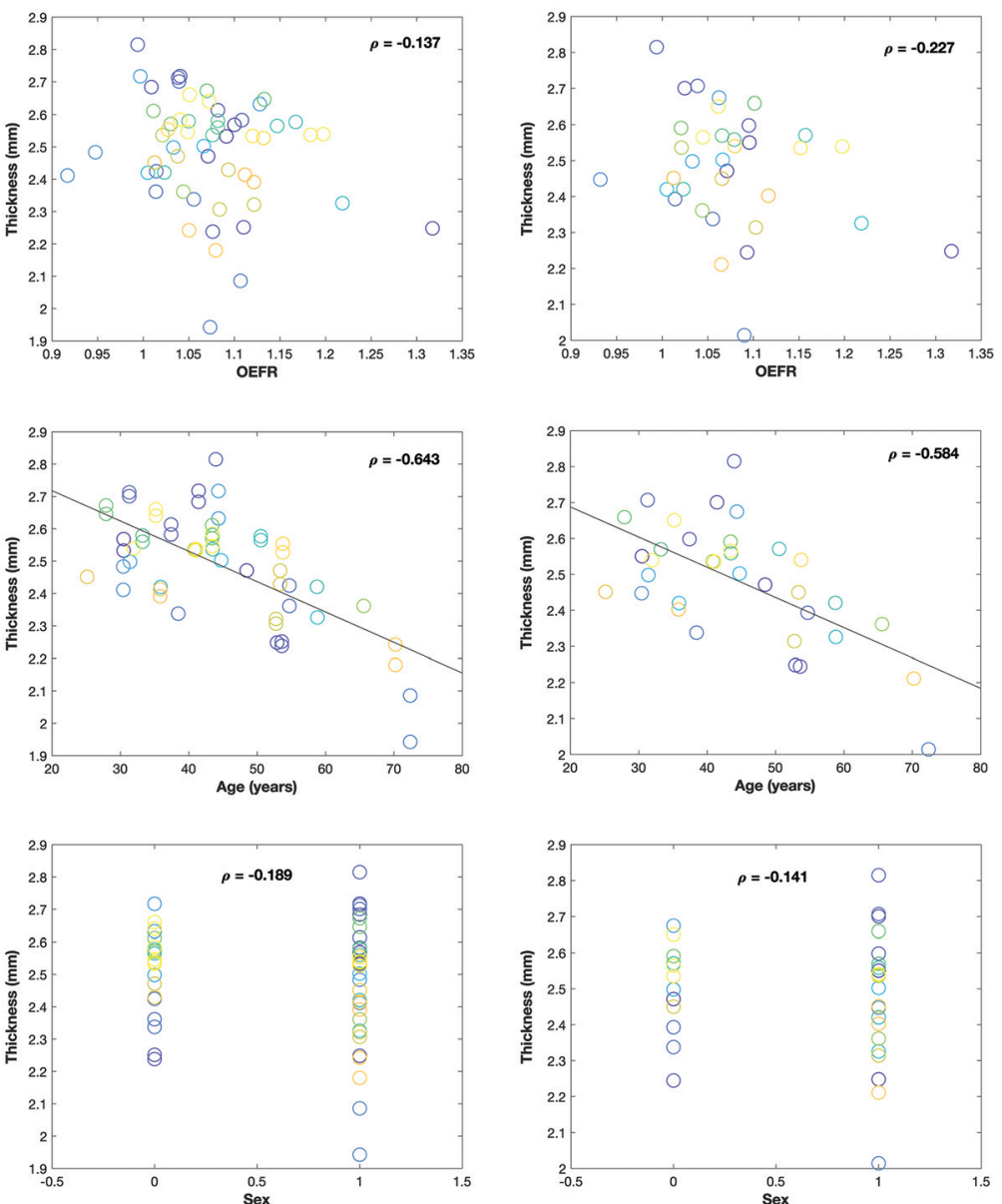

15
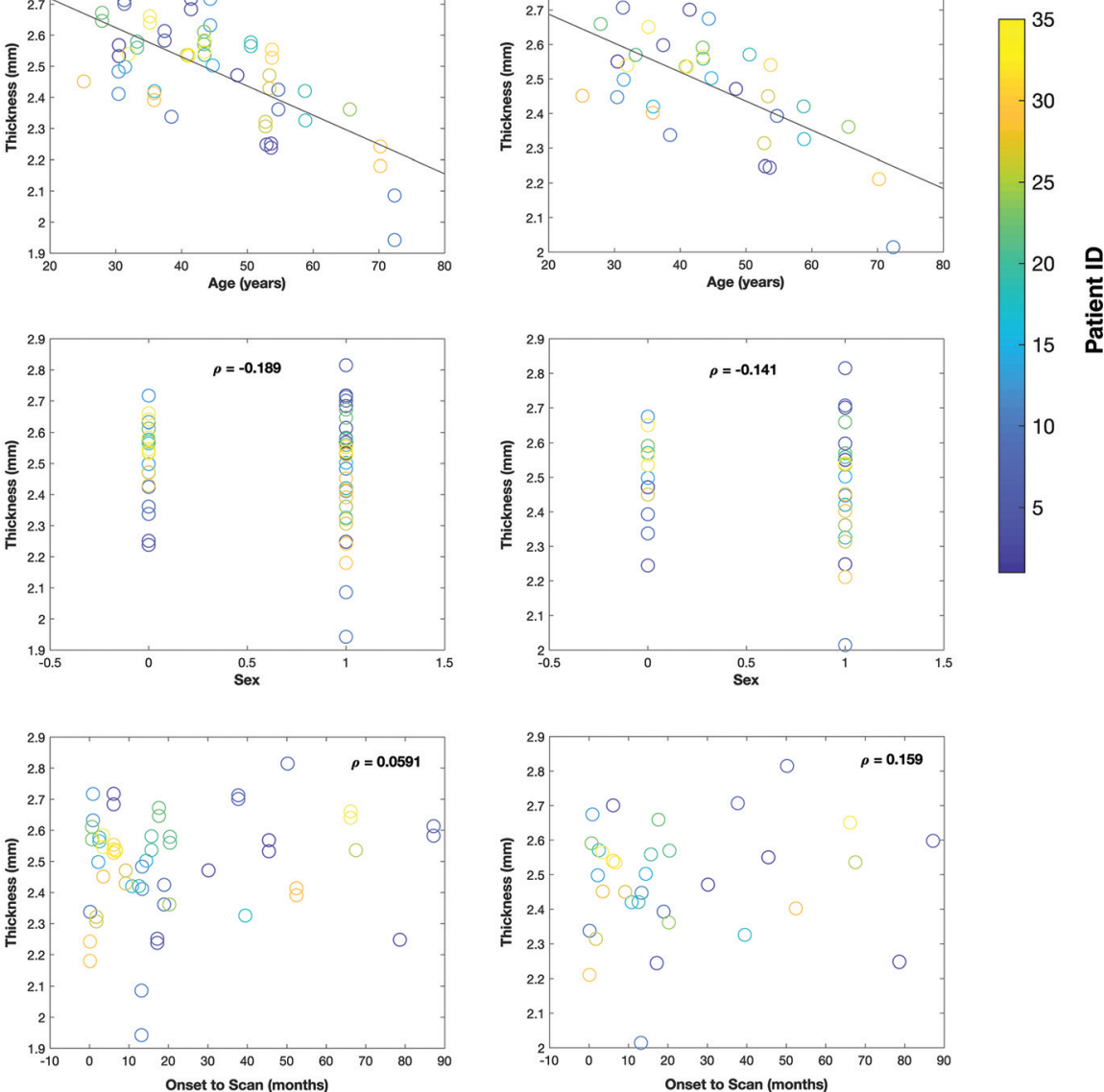

FIG 3. Plots of data arranged by granularity of measurements (FreeSurfer regions, hemispheres, patients) in columns and mappings of predictors (OEFR, age, sex, and time from symptom onset to scanning) of cortical thickness in rows. For sex, male $=0$ and female $=1$. The color map indicates patient identities. Lines of regression between model parameters and cortical thickness correspond to significant predictors. $\rho$ denotes the partial correlation coefficient between the plotted parameter and cortical thickness. The top-left panel excludes a data point with OEFR $=0.479$ and 4 data points with an OEFR of $>2$ to clarify the distribution of regional data with respect to OEFR and thickness. All plots exclude regions and hemispheres ipsilateral to infarctions or hemorrhages as detailed in the Materials and Methods.

phenomenon on the affected hemisphere and unaffected contralateral regions with each patient serving as his or her own control. ${ }^{21}$ In the present analysis, we included patients with bilateral and unilateral disease and idiopathic Moyamoya phenomenon. Third, we used mixed-effects models to examine OEFR and cortical thickness with explicit considerations for the hierarchy of FreeSurfer regions, hemispheres, and patient identities. We also selectively examined MCA territories, and included age, sex, and time between symptom onset and scanning in our models.

We used values of OEFR from Destrieux parcellation regions confined to MCA territories. Distal internal carotid occlusive disease was most likely to affect flow to these regions. We recognize that patients in this series may have had greater flow reduction to one or the other territory and some may have had posterior cerebral artery involvement. Averaging over entire hemispheres shifted the significance of regressions from OEFR and age to only age, a well-studied predictor for cortical thickness. ${ }^{20}$ Diagnosis and surgical treatment options for patients with Moyamoya phenomenon may benefit from FreeSurfer-based regional measurements of OEF, OEFR, or cortical thickness. 
Carotid disease and chronic hemodynamic impairment are associated with reduced cognitive function. Subclinical stroke represents the major potential confound for this association. ${ }^{22}$ In baseline data from the Randomized Evaluation of Carotid Occlusion and Neurocognition (RECON) study, Marshall et $\mathrm{al}^{23}$ reported a strong association between increased OEF and reduced baseline neurocognitive status. Patients with increased OEF on study entry were much more likely to have neurocognitive impairment than patients with a normal OEF.

Finally, there is good evidence that cortical thickness is dynamic. ${ }^{24,25}$ Increases in gray matter volume and cortical thickness have been reported with learning new functions. These changes can be measured within days and are postulated to reflect neuronal changes, such as spine and synapse turnover, ${ }^{26}$ given the time course. ${ }^{24}$ Changes in the microvasculature are possible (eg, reduced capillary density, vasoconstriction), but difficult to explain. The associations of cortical thickness with age that we observed are well-described and provide further evidence of the external validity of our methods and observations; however, associations of cortical thickness with sex are likely confounded by the predominance of women with Moyamoya phenomenon in our study cohort. ${ }^{11,20,27,28}$ The alternative explanations for reduced cortical thickness would generally not be reversible. These would include selective neuronal loss, submillimeter cortical infarctions, and white matter infarction leading to reduced cortical activity.

\section{CONCLUSIONS}

Our data support the hypothesis that chronic hemodynamic impairment measured by PET is regionally associated with reduced cortical thickness. The effects of regional chronic hemodynamic impairment are significant and comparable with age for explaining reduced cortical thickness; age is a well-studied predictor of cortical thickness in healthy adults. Given the evidence from other studies that cortical thickness decreases are reversible, it is possible that decreases reflect metabolic down-regulation that may, in turn, be associated with reversible cognitive impairment. Future studies that incorporate serial measurements of oxygen metabolism and neuropsychological testing will be required to test these hypotheses.

For enhanced reproducibility, analysis software, unit tests, and summary data are provided at https://github.com/jjleewustledu/ Hemodynamic-Impairment-Measured-by-PET-is-RegionallyAssociated-with-Cortical-Thickness-in-Moyamoya.

Disclosures: John J. Lee—RELATED: Grant: National Institutes of Health, Comments: Supported by National Institute of Neurological Disorders and Stroke RO1 NS051631. Support for the research imaging center where the MR imaging studies were performed was provided by 1 UL1 RR024992-01, 1 TL1 RR024995-01, and 1 KL2 RR 024994-01 from the National Center for Research Resources, a component of the National Institutes of Health, and the National Institutes of Health Roadmap for Medical Research and P30NS048056*. Joshua S. Shimony-UNRELATED: Expert Testimony: expert testimony in regard to the use of DTI in the diagnosis of traumatic brain injury; Grants/Grants Pending: National Institutes of Health *. Gregory R. ZipfelUNRELATED: Consultancy: Gerson Lehrman Group, Comments: consulting on a medical topic outside that discussed in the present article; Expert Testimony: Wais, Vogelstein, Forman and Offutt; Darby \& Gaza; Zeigler Cohen \& Koch; Hyde, Love, and Overby; Boyle Brasher LLC, Comments: expert witness for a variety of medical conditions; Grants/Grants Pending: National Institutes of Health. * ${ }^{*}$ Money paid to institution.

\section{REFERENCES}

1. Fierstra J, Maclean DB, Fisher JA, et al. Surgical revascularization reverses cerebral cortical thinning in patients with severe cerebrovascular steno-occlusive disease. Stroke 2011;42:1631-37 CrossRef Medline

2. Derdeyn CP, Grubb RL Jr, Powers WJ. Cerebral hemodynamic impairment: methods of measurement and association with stroke risk. Neurology 1999;53:251-51 CrossRef Medline

3. McHenry LC Jr, Fazekas JF, Sullivan JF. Cerebral hemodynamics of syncope. Am J Med Sci 1961;241:173-78 Medline

4. Derdeyn CP, Videen TO, Yundt KD, et al. Variability of cerebral blood volume and oxygen extraction: stages of cerebral haemodynamic impairment revisited. Brain 2002;125:595-607 CrossRef Medline

5. Powers WJ, Clarke WR, Grubb RL Jr, et al; CROSS Investigators. Extracranial-intracranial bypass surgery for stroke prevention in hemodynamic cerebral ischemia: the Carotid Occlusion Surgery Study randomized trial. JAMA 2011;306:1983-92 CrossRef Medline

6. Grubb RL Jr, Derdeyn CP, Fritsch SM, et al. Importance of hemodynamic factors in the prognosis of symptomatic carotid occlusion. JAMA 1998;280:1055-60 CrossRef Medline

7. Yamauchi H, Fukuyama H, Nagahama Y, et al. Significance of increased oxygen extraction fraction in five-year prognosis of major cerebral arterial occlusive diseases. J Nucl Med 1999;40:1992-98 Medline

8. Derdeyn CP, Videen TO, Simmons NR, et al. Count-based PET method for predicting ischemic stroke in patients with symptomatic carotid arterial occlusion. Radiology 1999;212:499-506 CrossRef Medline

9. Powers WJ. William M. Feinberg award for excellence in clinical stroke: hemodynamics and stroke risk in carotid artery occlusion. Stroke 2014;45:3123-28 CrossRef Medline

10. Jiang TT, Videen TO, Grubb RL Jr, et al. Cerebellum as the normal reference for the detection of increased cerebral oxygen extraction. J Cereb Blood Flow Metab 2010;30:1767-76 CrossRef Medline

11. Derdeyn CP, Zipfel GJ, Zazulia AR, et al. Baseline hemodynamic impairment and future stroke risk in adult idiopathic Moyamoya phenomenon: results of a prospective natural history study. Stroke 2017;48:894-99 CrossRef Medline

12. Dale AM, Fischl B, Sereno MI. Cortical surface-based analysis, I: segmentation and surface reconstruction. Neuroimage 1999;9: 179-94 CrossRef Medline

13. Fischl B, Sereno MI, Dale AM. Cortical surface-based analysis, II: inflation, flattening, and a surface-based coordinate system. $\mathrm{Neu}$ roimage 1999;9:195-207 CrossRef Medline

14. Fischl B. FreeSurfer. Neuroimage 2012;62:774-81 CrossRef Medline

15. Derdeyn CP, Videen TO, Grubb RL Jr, et al. Comparison of PET oxygen extraction fraction methods for the prediction of stroke risk. J Nucl Med 2001;42:1195-97 Medline

16. Karakatsanis N, Sakellios N, Tsantilas NX, et al. Comparative evaluation of two commercial PET scanners, ECAT EXACT HR + and Biograph 2, using GATE. Nuclear Instruments and Methods in Physics Research A 2006;569:368-72 CrossRef

17. Destrieux C, Fischl B, Dale A, et al. Automatic parcellation of human cortical gyri and sulci using standard anatomical nomenclature. Neuroimage 2010;53:1-15 CrossRef Medline

18. Smith SM, Jenkinson M, Woolrich MW, et al. Advances in functional and structural MR image analysis and implementation as FSL. Neuroimage 2004;23:S208-19 CrossRef Medline

19. van der Zwan A, Hillen B, Tulleken CAF, et al. Variability of the territories of the major cerebral arteries. J Neurosurg 1992;77: 927-40 CrossRef Medline

20. Salat DH, Buckner RL, Snyder AZ, et al. Thinning of the cerebral cortex in aging. Cereb Cortex 2004;14:721-30 CrossRef Medline

21. Fierstra J, Poublanc J, Han JS, et al. Steal physiology is spatially associated with cortical thinning. J Neurol Neurosurg Psychiatry 2010; 81:290-93 CrossRef Medline

22. Romero JR, Beiser A, Seshadri S, et al. Carotid artery atherosclerosis, 
MRI indices of brain ischemia, aging, and cognitive impairment: the Framingham Study. Stroke 2009;40:1590-96 CrossRef Medline

23. Marshall RS, Festa JR, Cheung YK, et al. Cerebral hemodynamics and cognitive impairment: baseline data from the RECON trial. Neurology 2012;78:250-55 CrossRef Medline

24. Driemeyer J, Boyke J, Gaser C, et al. Changes in gray matter induced by learning: revisited. PLoS One 2008;3:e2669 CrossRef Medline

25. Bermudez P, Lerch JP, Evans AC, et al. Neuroanatomical correlates of musicianship as revealed by cortical thickness and voxel-based morphometry. Cereb Cortex 2009;19:1583-96 CrossRef Medline
26. Trachtenberg JT, Chen BE, Knott GW, et al. Long-term in vivo imaging of experience-dependent synaptic plasticity in adult cortex. Nature 2002;420:788-94 CrossRef Medline

27. Gautam P, Cherbuin N, Sachdev PS, et al. Sex differences in cortical thickness in middle aged and early old-aged adults: personality and total health through life study. Neuroradiology 2013;55:697-707 CrossRef Medline

28. Fjell AM, Westlye LT, Amlien I, et al. High consistency of regional cortical thinning in aging across multiple samples. Cereb Cortex 2009;19:2001-12 CrossRef Medline 